\title{
Information is a key prerequisite for perceived relevance of patient reported outcome data (PRO data): A multicenter questionnaire study
}

\author{
Malene Kildemand ${ }^{1}$, Hanne Merete Lindegaard ${ }^{1}$, Mette Juel Rothmann ${ }^{2,3}$, Birgitte Nørgaard*4 \\ ${ }^{1}$ Department of Rheumatology, Odense University Hospital, Odense, Denmark \\ ${ }^{2}$ Department of Endocrinology and Rheumatology, Odense University Hospital, Odense, Denmark \\ ${ }^{3}$ Center for Innovative Medical Technology and Department of Clinical Research, University of Southern Denmark, Odense, \\ Denmark \\ ${ }^{4}$ Department of Public Health, University of Southern Denmark, Odense, Denmark
}

Received: December 12, 2018

Accepted: January 22, 2019

Online Published: March 18, 2019

DOI: $10.5430 /$ jnep.v9n6p107

URL: https://doi.org/10.5430/jnep.v9n6p107

\begin{abstract}
Objective: The aim of this study was to investigate the relevance of patient reported outcome data (PRO data) as assessed by arthritis patients in a Danish hospital setting.

Methods: The study was conducted as a multicenter questionnaire survey comprising patients with rheumatoid arthritis, ankylosing spondylitis, and psoriatic arthritis at three rheumatology outpatient clinics. Respondents with experience of reporting PRO data were recruited. The recruitment took place in March 2017.

Results: A total of 98 respondents were included. We found significant correlation between respondents' level of information about PRO data and the perceived relevance of PRO data questions. Remarkably, a third of the respondents stated that PRO data prepared neither themselves nor the healthcare professionals for the consultation, while $40 \%$ found that their PRO data responses were not used during consultations with healthcare professionals.

Conclusions: The respondents' assessment of the relevance of PRO data depended on the information offered to them. In recognition of its potential as a tool for patient involvement, the use of PRO data should be formally integrated in routine clinical care.
\end{abstract}

Key Words: PRO data, Patient reported outcome data, Patients' assessment, Questionnaire survey

\section{INTRODUCTION}

Patient reported outcome data (PRO data) are used to monitor patients' condition by examining the patients' own view on their symptoms, functional status and health related quality of life. The use of PRO data was originally developed for research purposes but has gained increasing impact on modern healthcare as a tool for enhancing patient involvement in treatment and care by using the patients' report of their own status to plan the treatment. ${ }^{[1,2]}$ Thus, repeatedly asking patients to respond to the same PRO questions regarding their health status enables the real time assessment of PRO data on chronic conditions, thereby adding to the traditional clinical, laboratory, and imaging measures of disease regarding e.g. disease activity and treatment choices. ${ }^{[1]}$ When patients

\footnotetext{
*Correspondence: Birgitte Nørgaard; Email: binorgaard@ @ealth.sdu.dk; Address: Department of Public Health, University of Southern Denmark, J.B. Winsløws Vej 9B, DK-5000 Odense, Denmark.
} 
assess their treatment and care and the data are compared with healthcare professionals' assessments, the results may serve as a basis for joint decision-making on future management, for example to improve communication between patients and healthcare professionals and to focus on issues of particular relevance to the individual patient. ${ }^{[1,3]}$ By contributing to patient involvement through tailoring treatment and care, PRO data can improve individual care. Recent evidence that both patients and healthcare systems benefit from involving patients in decisions on treatment choices and care is of particular interest. As patients learn to protect and improve their health, the utilization of healthcare services may be improved. ${ }^{[4,5]}$

Chronic inflammatory joint diseases have a major impact on quality of life and daily functioning, both in leisure and professional activities. ${ }^{[2]}$ PRO data may therefore play an important role in the management of such diseases by enabling a stronger focus on the patient's perspective. It should be recognized that only the patients themselves can offer a truly relevant perspective on the outcome of the treatment and their current wellbeing, including global health-defining variables such as fatigue and sleep. ${ }^{[6]}$ Rheumatology has a long tradition for using PRO data in research, routine clinical care, and clinical trials. ${ }^{[7]}$ In Denmark, PRO data are systematically collected from patients with rheumatic diseases and stored in the nationwide DANBIO (full name, not an abbreviation) registry. A web-based questionnaire is used for the data collection, with patients entering data on touch screens in waiting areas before consultation with the physician or the nurse. ${ }^{[8]}$ The PRO data collected in DANBIO include the visual analogue scales (VAS) for pain, fatigue, and the patient's global assessment (PGA). In addition, the patients completed the EuroQol-5D questionnaire (EQ-5D).

Disease-specific PRO data were furthermore elicited via the Health Assessment Questionnaire (HAQ) for patients with rheumatoid arthritis, and the Bath Ankylosing Spondylitis Disease Activity Index (BASDAI) and the Bath Ankylosing Spondylitis Function Index (BASFI) for patients with ankylosing spondylitis or psoriatic arthritis. ${ }^{[8]}$

Despite growing interest in PRO data and patient involvement, research into the patients' perspectives on PRO data utility has been limited. It has focused mostly on the format of PRO data, whereas the patients' perspective of functionality and feedback is little investigated, as shown in a synthesis by Greenhalgh et al. ${ }^{[9]}$ Because PRO data have the potential to improve patient treatment and care, the investigation of PRO data from the perspective of the patients is highly relevant. The aim of this study was to investigate the relevance of PRO data as assessed by arthritis patients in a Danish rheumatology clinic setting.

\section{METHODS}

\subsection{Design}

We conducted a descriptive cross-sectional questionnaire survey of patients from three Danish rheumatology outpatient clinics in which PRO data were systematically collected.

\subsection{Setting and respondents}

Data collection took place during March 2017 and was conducted by the first author (MK). Patients were included consecutively in the clinics' waiting areas immediately after they had entered their PRO data on touch screens. Eligible participants had to be at least 18 years of age and able to understand Danish. Patients with cognitive impairment were excluded.

\subsection{The questionnaire}

We designed a questionnaire covering six overarching domains in 27 items. The construction of the questionnaire was based on a systematic literature search using the keywords "PRO data", "patient involvement", "rheumatoid arthritis", and their synonyms. A total of 38 studies were selected for further reading, five of which were used in formulating the questions. ${ }^{[8,10-13]}$ Three reports investigating PRO data from a Danish context were used. ${ }^{[14-16]}$

The first domain included seven items eliciting sociodemographic information. In the second domain, five items assessed the manner of informing patients about the use of PRO data. The six items of the third domain covered physical and technical difficulties as well as the environment in which the PRO data were entered, concerning privacy. The engagement of the respondents toward the PRO data was investigated via the three items of the fourth domain; the five items of the fifth domain assessed the patient's perception of the relevance of the PRO questions and their application during consultations. The sixth domain provided space for any additional comments. The response options for questions 8-26 were "not at all", "to a lesser extent", "to some extent", "to a higher extent", or "don't remember". A few items had numerical or descriptive response options.

Responses were entered into an Apple iPad tablet computer.

\subsection{Validation and pilot-testing}

To ensure that the questions were comprehensible, the questionnaire was face-validated using the think aloud method in a representative group of four patients (two men and two women). It was additionally validated by a group of healthcare professionals including three registered nurses (RNs), two physicians, and a professor of medicine, all of whom had experience from rheumatology and the use of PRO data. 
A pilot test by four patients focused specifically on the functioning of the tablet computer. No changes were made based on the validations.

\subsection{Data analysis}

The results are described by proportions and analyzed by chi-square and Fisher's exact test using Stata, version 14 (StataCorp. 2015. Stata Statistical Software: Release 14. College Station, TX: StataCorp LP).

\subsection{Ethical considerations}

The patients received oral and written information about the purpose of the study. They were furthermore informed that their privacy was protected, that participation was voluntary, and that withdrawal would have no consequences for their therapy. The patients were informed that by responding to the questionnaire, they accepted inclusion in the study. A unique identification number used for data entry secured the respondents' anonymity, thus precluding their identification. The study was approved by the relevant heads of department; no further ethical approval was required according to Danish legislation.

\section{RESULTS}

\subsection{Respondents}

The inclusion criteria were met by 105 patients during the data collection period. Five were excluded due to incomplete answers and two were excluded as they were not attached to any of the three clinics chosen for this study. Hence, 98 respondents were included (93.3\% response rate).

The sociodemographic characteristics of respondents are shown in Table 1. Their mean age was 54.6 years, ranging from 20 to 87 years. The majority of the respondents had been attending the clinic for between one and four years $(35.7 \%)$.

\subsection{Information about PRO data}

When asked to what extent they had been informed about the use of their PRO data, one third of the respondents (29.6\%) stated that they had received no information ("none at all") or limited information ("to a lesser extent"). While 59.2\% reported having received oral information about the PRO data, only $8.2 \%$ recalled having received written information.

A significant positive association was found between the number of years of affiliation with the clinic and being given written information. Significantly more respondents who had attended the clinic for at least five years stated having received written information, compared to respondents with a shorter follow-up time ( $p=.001)$ (see Table 2).

Published by Sciedu Press
Table 1. Characteristics of respondents

\begin{tabular}{ll}
\hline & $\%(\mathbf{n})$ \\
\hline Fender & $70.4(69)$ \\
Male & $29.6(29)$ \\
Age (years) & \\
$18-39$ & $17.3(17)$ \\
$40-59$ & $35.7(35)$ \\
$60-79$ & $44.9(44)$ \\
Over 80 & $2(2)$ \\
Diagnosis & \\
Rheumatoid arthritis & $65.3(64)$ \\
Psoriatic arthritis & $14.3(14)$ \\
Ankylosing spondylitis & $15.3(15)$ \\
Other & $3.1(3)$ \\
Don't know & $2(2)$ \\
Duration of affiliation with outpatient clinic & \\
$<1$ year & $15.3(15)$ \\
1-4 years & $35.7(35)$ \\
5-7 years 8 years & $19.4(19)$ \\
\hline
\end{tabular}

\subsection{Physical and technical difficulties}

The majority of the respondents $(75.5 \%)$ indicated that they had never experienced physical difficulties when reporting PRO data on the touch screens. In contrast, technical difficulty had been experienced by $52 \%(n=51)$, who stated that this "always", "sometimes", or "rarely" happened. Five of the 51 stated that the technical difficulties had a negative influence on their PRO data responses, as they were distracted by the disturbing circumstances.

We found no significant differences the responses of men and women regarding the experience of technical difficulties ( $p$ $=.35$ ); no association was found between age and technical difficulties $(p=.39)$ (see Table 2).

\subsection{Context}

A total of $79.6 \%$ of the participants reported that their PRO data responses had not been influenced by the fact that they had had to enter the data while in waiting areas with other people around them (e.g., patients, healthcare professionals, relatives). On the other hand, 58.2\% expressed an interest in ("to some degree/to a higher degree") being given the opportunity to respond to the PRO questionnaire from their home. As this was most prevalent among respondents aged between 20 and 59 years, a significant association between age and interest in responding to the PRO questionnaire at home was observed $(p=.006)$. Younger respondents were significantly more positive toward responding from home. 


\subsection{Engagement of respondents}

Three out of 4 respondents (74.5\%) saw it as rather important to make an effort when responding to the PRO questionnaire $(\mathrm{n}=73)$ (responding "to a higher extent"). Of these, $41.1 \%$ $(\mathrm{n}=30)$ indicated that they had been informed "to a high extent" about the use of PRO data.

\subsection{Relevance}

Regardless of their diagnosis, a large majority of respondents $(81.6 \%)$ reported that they found the PRO data questions relevant.

A significant positive association was found between the perceived level of information regarding the use of PRO data and the perceived relevance of the PRO data questions ( $p=$ .002) (see Table 2).

Asked if the PRO data questions had prepared them well for their consultations with the physician or the nurse, nearly one third of the participants responded either "don't know" or "to a lesser degree". In response to the question whether they found that the PRO data had prepared the physician or nurse for the consultation, similar answers were obtained. Likewise, $40 \%$ reported either "don't know" or "not at all or to a lesser degree" when asked whether their PRO data answers had been used in the consultation.

\subsection{Insight into own health}

A total of $42.9 \%$ of the respondents said either "don't know", "not at all", or "to a lesser extent" when asked whether the PRO data questions had given them insight into their own health status. There was no significant association between the respondents' diagnoses and whether they assessed the PRO data questions to have given them insight into own health or not $(p=.262)$.

Table 2. Patients' assessment of PRO data

\begin{tabular}{|c|c|c|c|}
\hline & Positive \% (n) & Negative \% (n) & p-value \\
\hline \multicolumn{4}{|c|}{ Association between receiving written information and duration of the respondents' affiliation with outpatient clinic } \\
\hline$<1-4$ years & $8.3(3)$ & $91.7(33)$ & \multirow{2}{*}{$p=.001$} \\
\hline 5- $>8$ years & $15.1(5)$ & $84.8(28)$ & \\
\hline \multicolumn{4}{|c|}{ Association between technical difficulties and gender and age } \\
\hline Men & $44.8(13)$ & $55.2(16)$ & \multirow{2}{*}{$p=.35$} \\
\hline Women & $55.1(38)$ & $44.9(31)$ & \\
\hline 20-59 years & $48.1(25)$ & $51.9(27)$ & \multirow{2}{*}{$p=.39$} \\
\hline $60<$ & $56.5(26)$ & $43.5(20)$ & \\
\hline \multicolumn{4}{|c|}{ Association between wish for responding to questionnaire from home and age } \\
\hline 20-59 years & $70.2(33)$ & $29.8(14)$ & \multirow[t]{2}{*}{$p=.006$} \\
\hline $60->80$ years & $55.8(24)$ & $44.2(19)$ & \\
\hline \multicolumn{4}{|c|}{$\begin{array}{l}\text { Association between perceived relevance of questions combined with the degree to which respondent were informed about } \\
\text { use of responses }\end{array}$} \\
\hline Informed to some or to higher degree & $50.8(32)$ & $46(29)$ & \multirow{2}{*}{$p=.002$} \\
\hline No information or informed to a lesser degree & $17.2(5)$ & $79.3(23)$ & \\
\hline
\end{tabular}

\section{Discussion}

Approximately one third of the respondents reported that the information received regarding the use of their PRO data had been nonexistent ("not at all") or limited ("to a lesser extent"). We found a significant association between the perceived level of information about the PRO data and the perceived relevance of the PRO data questions.

A study by Van Der Wees et al. has investigated the optimal development and selection of PRO data for experimental and clinical use. The authors concluded that PRO data must be considered relevant for the individual patient in order for them to continue supplying PRO data. ${ }^{[17]}$ Another study concluded that a lack of knowledge among healthcare professionals about the use of PRO data presents an obstacle to their successful implementation in routine clinical care. Learning how PRO data can be exploited would help healthcare professionals optimize patient involvement in decision-making. ${ }^{[18]}$ However, we found no studies investigating the need for, or effect of, information regarding PRO data to patients in an everyday clinical setting. In their investigation of patients' assessment of PRO data gleaned using the Health Assessment Questionnaire (HAQ), Ebbevi et al. found that patients with rheumatoid arthritis were critical of HAQ for its failure to 
embrace aspects of extreme relevance to them. ${ }^{[19]}$ These results contradict our findings as our respondents, irrespective of their diagnosis, found the PRO data questions (including those of the HAQ) to be relevant. This discrepancy could at least in part be attributable to our simultaneous use of multiple PRO data measures, which may have compensated for the shortcomings of HAQ scoring alone.

The majority of our study respondents stated that reporting PRO data in the waiting area at the clinic had no influence on their answers. However, it may be challenging to find privacy in waiting areas; a problem which could be solved by offering patients the opportunity to fill the PRO questionnaire at home, as suggested by Cella et al. ${ }^{[10]}$ In Denmark, the dissemination of computers and smartphones is generally high, with $85 \%$ of the population daily or near-daily internet users. ${ }^{[20]}$ However, it should be noted that self-evaluated IT competences decrease with age, and $40 \%$ of the population aged 75 to 89 years does not feel comfortable using the internet. ${ }^{[20]}$ This is probably reflected in our finding that 20 to 59-year-old respondents were significantly more interested in responding to the PRO questionnaire at home, compared to those of 60 years or older.

The use of PRO data is a potentially relevant tool for involving arthritis patients in their own care. It may allow for treatment decisions to be based not only on clinical, biochemical, and imaging findings, but also on patients' systematic reporting on which effect their symptoms and treatment have on the patients' daily life. However, in a survey conducted by the Danish Knowledge Center for User Involvement in Health Care (The Danish acronym; VIBIS), the nurse and physician respondents said that even though they found that patient involvement contributes to increased patient satisfaction, only one in four of them actually involved patients in treatment and care decisions. ${ }^{[21]}$

Our study shows that almost one third of the respondents indicated that their PRO data responses did not appear to prepare the healthcare professionals for consultations. Forty percent of them reported that their PRO data answers were not used in the consultation. This is in line with results from the VIBIS survey showing that patients' knowledge is poorly utilized in the consultations. ${ }^{[21]}$ When medical records and PRO data are included in the consultations it can be considered as shared decision-making. Following this mindset, we advise healthcare professionals to regard PRO data as a useful adjunct to the biomedical perspective. ${ }^{[9]}$ Shared decision-making may contribute to better health behaviors and increased knowledge, understanding of and compliance with the mutually chosen treatment. ${ }^{[4]}$ Nota et al. found that, while acknowledging the physicians' expertise, patients with rheumatoid arthritis expressed a wish to be involved in decisions about their treatment as they strive to gain control of their symptoms and that their treatment be incorporated into their daily lives. ${ }^{[11]}$ Conversely, a significant disadvantage of shared decision-making is the possibility that patients manipulate their PRO data responses. Patients may thus report stronger pain than experienced in order to receive a desired treatment, with the risk that their treatment does not fulfill current standards of care and the loss of confidence between patient and healthcare professionals, ${ }^{[9]}$ dilemmas that are also addressed by our study. Even though the respondents were not asked directly whether they manipulated their answers, we did ask them if they found it important to make an effort when responding to the questions. A total of $93.9 \%$ stated that they did this "to some" or a "to higher extent". We take this as indication that the majority of the respondents gave some consideration to their answers.

\section{Strengths and limitations}

The strength of this study may be limited by the fact that the patients were not involved in the preparation of the questionnaire, which might have given them a say on the relevance of its questions. However, the patients were involved in the face validation of the questions using the think aloud method, and afterwards pilot tested the questionnaire, which we consider a strength regarding content validation.

The respondents were considered representative of the population under investigation. Women and respondents with rheumatoid arthritis were overrepresented, reflecting the gender distribution and the prevalence of autoimmune diseases. ${ }^{[22]}$

Observing a degree of caution, we believe that our results can be transferred to other rheumatology departments using PRO data in treatment and care. Nevertheless, a study with a larger sample size and the inclusion of more centers would be able to strengthen our conclusion.

\section{Conclusion}

This study shows that being offered information about PRO data and their application is important to the respondents in their assessment of PRO data. We found a significant association between the level of information and the patient perceived relevance of the use of PRO data.

Notably, more than one third of the respondents reported that PRO data had not prepared them for their consultation and that it was not their experience that their PRO data answers had prepared the healthcare professionals for the consultation. Forty percent of the participants assessed that their PRO data answers were not used in the consultation. 
Our study has uncovered a neglected aspect of the patients' assessment of PRO data. While PRO data may offer a valuable adjunct to current disease indicators by strengthening the patient perspective, increased attention is required to unfold its full potential. Further research on larger samples is needed to investigate patients' experience with PRO data in order to produce more solid and generalizable results.

\section{Conflicts of Interest Disclosure}

The authors declare that there is no conflict of interest.

\section{REFERENCES}

[1] Black N. Patient reported outcome measures could help transform healthcare. British Medical Journal. 2013.

[2] Nikiphorou E, Radner H, Chatzidionysiov K, et al. Patient global assessment in measuring disease activity in rheumatoid arthritis: a review of the literature. Arthritis Research \& Therapy. 2016; 18(251).

[3] Chen J, Ou L, Hollis SJ. A systematic review of the impact of routine collection of patient reported outcome measures on patients, providers and health organisations in an oncologic setting. BMC Health Services Research. 2013.

[4] Coulter A. Patient Engagement-What Works? Journal of Ambulatory Care Management. 2012; 35(2): 80-9. PMid:22415281 https://doi.org/10.1097/JAC.0b013e318249e0fd

[5] Ocloo J, Matthews R. From tokenism to empowerment: progressing patient and public involvement in healthcare improvement. BMJ Quality \& Safety. 2016; 25: 626-32. PMid:26993640 https : //doi.org/10.1136/bmjqs-2015-004839

[6] El Miedany Y. PROMs in inflammatory arthritis: moving from static to dynamic. Clinical Rheumatology. 2013; 32(6): 735-42. PMid:23572036 https ://doi.org/10.1007/s10067-013-222 8-0

[7] Callahan LF. The history of Patient-Reported Outcomes in rheumatology. Rheumatic Disease Clinics of North America. 2016; 42(2).

[8] Ibfelt EH, Jensen DV, Hetland ML. The Danish nationwide clinical register for patients with rheumatoid arthritis: DANBIO. Clinical Epidemiology. 2016; 8: 737-42. PMid:27822121 https://doi.or g/10.2147/CLEP. S99490

[9] Greenhalgh J, Dalkin S, Gooding K, et al. Functionality and feedback: A realist synthesis of the collation, interpretation and utilisation of patient-reported outcome measures data to improve patient care. Health Service And Delivery Research. 2017; 5(2).

[10] Cella D, Hahn EA, Jensen SE, et al. Patient-Reported Outcomes in Performance Measurement: RTI Press; 2015.

[11] Nota I, Drossaert CHC, Taal E, et al. Arthritis patients' motives for (not) wanting to be involved in medical decision-making and the factors that hinder or promote patient involvement. Clinical Rheumatology. 2016; 35: 1225-35. PMid:25392118 https ://doi .org/10 $.1007 / \mathrm{s} 10067-014-2820-\mathrm{y}$
[12] Richter JG, Becker A, Koch T, et al. Self-assessments of patients via Tablet PC in routine patient care: comparison with standardized paper questionnaires. Annals of the Rheumatic Diseases. 2008; 67: 1739-41. PMid:18647853 https://doi.org/10.1136/ard. 2008. 090209

[13] Salaffi F, Gasparini S, Grassi W. The use of touch-screen technology for the collection of patient-reported outcome data in rheumatoid arthritis: comparison with standardized paper questionnaires. Clinical and Experimental Rheumatology. 2009; 27: 459-68. PMid:19604439

[14] Pedersen AB, Mikkelsen EM, Johnsen SP, et al. User-guide for implementation and use of patient-reported outcome data in clinical quality databases. [report in Danish] Aarhus; 2015.

[15] Danish Cancer Society. Clinical use of Patient Reported Outcome Measures (PROM): An evaluation report. [report in Danish] Copenhagen; 2016.

[16] VIBIS. Program PRO. The use of PRO-data in quality improvement of health care in the Danish healthcare system. Guidelines and evidence. [report in Danish] Copenhagen; 2016.

[17] Van Der Wees P, Nijhuis-Van Der Sanden M, Ayanian J, et al. Integrating the use of Patient-Reported Outcomes for both clinical pratice and performance measurement: Views of experts from 3 countries. The Milbank Quarterly. 2014; 92(4): 754-75. PMid:25492603 https ://doi.org/10.1111/1468-0009.12091

[18] Santana MJ, Haverman L, Absolom K, et al. Training clinicians in how to use patient-reported outcome measures in routine clinical practice. Quality of Life Research. 2015; 24: 1707-18. PMid:25589231 https://doi.org/10.1007/s11136-014-0903-5

[19] Ebbevi D, Essén A, Forsberg HH. Persons with rheumatoid arthritis challenge the relevance of the health assessment questionnaire: a qualitative study of patient perception. BMC Musculoskeletal Disorders. 2017; 18(189).

[20] Tassy A. The use of IT in the population. [report in Danish] Copenhagen: Statistics Denmark; 2016.

[21] VIBIS. Doctors and nurses' understanding of patient involvement-A survey among employees in hospitals. [resport in Danish] Copenhagen; 2014.

[22] Oliver JE, Silman AJ. Why are women predisposed to autoimmune rheumatic diseases? Arthritis Research \& Therapy. 2009; 11(5). 